\title{
Near-field light distributions propagated from human corneas: determination of relevant patterns
}

\author{
D. MAS, J. PEREZ, C. VAZQUEZ, C. HERNANDEZ and \\ C. ILLUECA*
}

Department Interuniversitari d’Òptica, Universitat d'Alacant, PO Box 99, E-03080, Alacant, Spain

(Received 23 January 2002; revision received 11 June 2002)

\begin{abstract}
A procedure to determine the best image range of distances that can be obtained from an aphakic eye is presented. Corneal surfaces are calculated from videokeratographic data. Light distributions at different distances inside the eye are calculated with a near-field Fresnel diffraction algorithm. These patterns are evaluated using three different quality functions which quantify the appearance of the energy distributions in each plane. Local extremes in those functions will serve to locate relevant distributions as Sturm focal planes and even the best image plane which is affordable by the cornea. The study is applied to strongly aberrated corneas.
\end{abstract}

\section{Introduction}

Evaluation of image quality of classical or holographic optical systems is a topic in geometrical and diffractive optics. In the case of the human eye, this evaluation becomes essential for the quality of life.

Among all the different parts of the eye, the most important one is the cornea. This surface is the interface between the air and the inner media inside the ocular globe, and thus it is responsible for the major part of the refractive power of the whole eye. As a consequence of this, it must have an important effect over the final image quality [1]. Aberrations of the cornea are modified by the internal ocular surfaces [2] to produce the final retinal image. Therefore, only measuring the anterior corneal aberrations seems not to be the most adequate procedure to determine the overall image quality in the eye. Despite this, the cornea is the main refracting element in the eye, and it may be considered as the major contributor to ocular aberrations. Moreover, this will certainly be the case in an abnormally aberrated cornea such as those affected by keratoconus or after refractive surgery [3].

Independently of the considered refractive surface, in general, two main strategies are followed in order to determine the quality of an optical system: the first consists of a purely geometric analysis which can be performed by calculating impact diagrams of light rays or studying the aberration function. With impact diagrams we examine the intersection points of rays leaving the

* Corresponding author: Prof. Carlos Illueca, phone: $(+34) 9659035$ 10; fax: (+ 34$) 96$ 59034 64; e-mail: illueca@ua.es 
optical system with a plane perpendicular to the axis at a certain distance. Dispersion of impact points will help to determine the performance of the particular system under study. The main drawback that appears with this technique is related to the amount of calculations which require a simple numerical analysis. The number of floating point operations which are required by a computer simulation increases dramatically with the number of rays. This limits the study to a few rays, typically below $50 \times 50$. Another more subtle problem is that at the exit plane, the sample distribution is not uniform and this impedes further the numerical processing of the data. In any case, the information that can be obtained with only spot-diagrams is always qualitative.

Analysis of optical systems has also been done through Zernike polynomials [4]. With these polynomials, it is possible to separate and classify the different irregularities that affect an imaging system. Among others, the main advantage of these polynomials is that adequate choice of the basis permits the description of any order aberrations, including Seidel aberrations [1]. Zernike polynomials also allow the obtention of the modulation transfer function of the cornea and how this function is affected by the different irregularities. In the case of the human cornea, Zernike decomposition appears to be unnecessarily sophisticated. In some cases the form of the surface is not as important as the propagation patterns which it generates. For example, one may be interested in the light distribution at an image plane or in how a test image evolutes after passing the cornea. In these cases a polynomic description of the surface may be useful but not absolutely necessary, and may be wasteful of computation time.

A second strategy which can be used for the study of optical quality of an imaging system deals with Fourier optics. The point spread function (PSF) or its Fourier transform, and the modulation transfer function (MTF) are powerful tools that are commonly used in optical testing. Usually, when parallel beams are used, the PSF is taken as the image distribution located at the focal plane of the system [5]. For rotationally symmetric surfaces this plane will coincide with the best image plane and this is approximately the case of normal corneas. On the other hand, when one is interested in non-uniform surfaces (i.e. astigmatic or highly deformed corneas) the focal plane is not well defined. In these cases it seems more interesting to look at a range of distances and determine the positions of distinctive image planes such as Sturm focal planes or the best image plane, where a symmetric peak is obtained.

Searching for an appropriate image plane may be of great importance in some cases. Let us consider one patient who suffers from a corneal malformation. Before performing any surgical process on the cornea or implanting a special intraocular lens it would be convenient to analyse the image formation process for a set of distances. This will quickly permit an intuitive analysis of the aberrated wavefront as it propagates through the different ocular media at any considered distance. This information can be used prior to an exhaustive and time consuming complete mathematical characterization of the cornea through Zernike polynomials.

Prior to analysing the optical quality of the cornea, some considerations must be taken into account. Consideration of the optical performance of the human eye usually leads to the correction or the compensation of possible defects which could be found. As we said before, the cornea is, perhaps, the most important part of the eye, but it is not the only one responsible for all image deformations which could appear in the final retinal image. Two main contributors exist for this imaging 
process: the cornea and the lens. The second element can vary its shape and power depending on the object position. Furthermore, it may introduce several aberrations that may modify the image produced by the cornea. In fact, in young normal eyes, the lens tends to partially compensate the aberrations introduced by the cornea [6]. Unfortunately, since mathematical modelling and analysis of the crystalline lens may be very complicated, we will limit our study to theoretical aphakic eyes, i.e. we will consider real corneas without any additional refractive element between this surface and the retina.

Having these considerations in mind, let us consider first regular corneas. In this case, a parallel beam striking the surface would propagate and concentrate at the focus. Thus, the best image plane can be clearly localized for a set of propagated patterns. In the case of deformed corneas the focal plane is not well defined. Thus, every plane must be analysed in order to determine a good image range of distances and, in the cases where it is possible, localize the best image plane.

Although different propagation patterns are available, it would take a lot of time to visualize all of them in order to find any plane of interest. Thus, every distribution will be characterized by three parameters which quantize the appearance of the image. These functions can be calculated for a range of distances by identifying every image plane with a quality factor, and consequently, image planes at a range of distances can be quickly analysed.

It will be advantageous for the evaluation process to be performed in real time. The bottleneck of the whole process will be the evaluation of the propagation patterns. In order to avoid further delay in the evaluation process, we propose three different simple parameters that will provide useful information at low computational cost.

One of the parameters that will be used here is the height of the energy peak. Ideally, the best image plane corresponding to a collimated beam source will be a delta function located at the focal plane. In a more realistic case, this image will be a peak function. The sharper and higher the peak is, the better will be the image obtained, and thus the better the quality of the refractive surface. In some sense this parameter is similar to the Strehl ratio, defined as the ratio of the irradiance values at the centre of the image plane with and without aberrations [7]. In our case, we have not done any a priori estimation of the non-aberrating pupil function. Hence, we have preferred to use another normalization for characterizing the peak value.

Another parameter which one must also consider is the symmetry of the peak. In general, corneas are almost symmetric, and thus, the image in the focal plane is a sharp and symmetric peak. For astigmatic corneas, the best image point is located between the two Sturm foci, and this will not coincide with the highest energy concentration but with a symmetric peak.

Low energy dispersion is also a quality criterion that identifies a narrow peak. We will measure this parameter through an entropy function. In digital information processing, the entropy concept represents an efficient use of the dynamic range of the data. In this sense, random light distributions present high levels of entropy while highly concentrated distributions will minimize this parameter [8]. In our case, entropy should be interpreted as a property of the way in which energy is distributed on an intensity image plane and, in this sense, entropy provides a measure of the aberrations in the considered planes. 
With all these three quality criterions, one will be able to find a range of distances where the best image plane produced by a cornea-in the sense of optimum energy distribution-could be located. For not very irregular corneas one can even find a best image plane which may not necessarily coincide with the paraxial focal plane of the cornea.

Fast determination of the best image range produced by the cornea through the three parameters defined here may be useful for refining the ablation process in surgical interventions or even designing new intra-ocular lenses. As an example of application, it can be used for defining the focal planes of a multifocal intra-ocular lens and determining the quality of the image planes provided by such an element.

Following the structure just outlined, in section 2 we will extend modelling the cornea as a phase object. In this paper, the surface will be reconstructed from the data obtained with a videokeratograph $[1,9]$, and then it will be modelled as a phase surface [10]. Propagation from the cornea to the distances of interest will be accomplished with a Fresnel propagation algorithm for short distances $[11,12]$. In section 3, the algorithm for calculating near-field light distributions will be briefly explained. The quality parameters that have been introduced above will be detailed in section 4 and advantages and disadvantages of these criteria will be discussed. In section 5 , these functions will be used to determine the best image range for several aphakic eyes. The performance of the method here proposed will be demonstrated by applying it to healthy and deformed corneas.

\section{Corneal maps and phase surfaces}

In recent years, videokeratoscopes have found widespread application in keratoconus screening, contact lens fitting and to pre- and post-operative corneal refractive evaluation. Most of these devices are based upon Placido disc set-ups, although alternative technologies exist.

The geometrical optics of a spherical reflecting surface is the basis for keratoscopy. In [13], the authors give details about the calculation of height corneal maps from videokeratographic data. Basically, keratographic data provide maps in polar coordinates $(\rho, \theta)$, where axial radii $R$ are codified in colour. From these data, the sagittal depth for each meridian can be estimated as

$$
g(\rho, \theta)=\int_{0}^{\rho} \frac{\rho^{\prime}}{\left[R^{2}\left(\rho^{\prime}\right)-\rho^{\prime 2}\right]^{1 / 2}} \mathrm{~d} \rho^{\prime} .
$$

Height maps can be interpreted as thickness functions measured from a reference plane. Let us consider a spherical diopter of radius $r$ limited by a pupil of diameter $D_{\mathrm{p}}$, as shown in figure 1 . The distance between the vertex of the diopter and the plane containing the pupil will be denoted here as $\Delta_{0}$. If this distance is small enough $\left(\Delta_{0} \ll r\right)$, we can consider that a particular point of a wavefront entering the diopter at coordinates $(x, y)$ will reach this plane at the same coordinates, but delayed by an amount proportional to the covered optical path $n \Delta(x, y)$. This is an extrapolation of the usual thin lens approximation [10].

Following the ideas described in the previous paragraph, the total phase delay suffered by a monochromatic wave at coordinates $(x, y)$ may be written:

$$
\phi(x, y)=\frac{2 \pi}{\lambda_{0}} n \Delta(x, y)+\frac{2 \pi}{\lambda_{0}}\left[\Delta_{0}-\Delta(x, y)\right]
$$




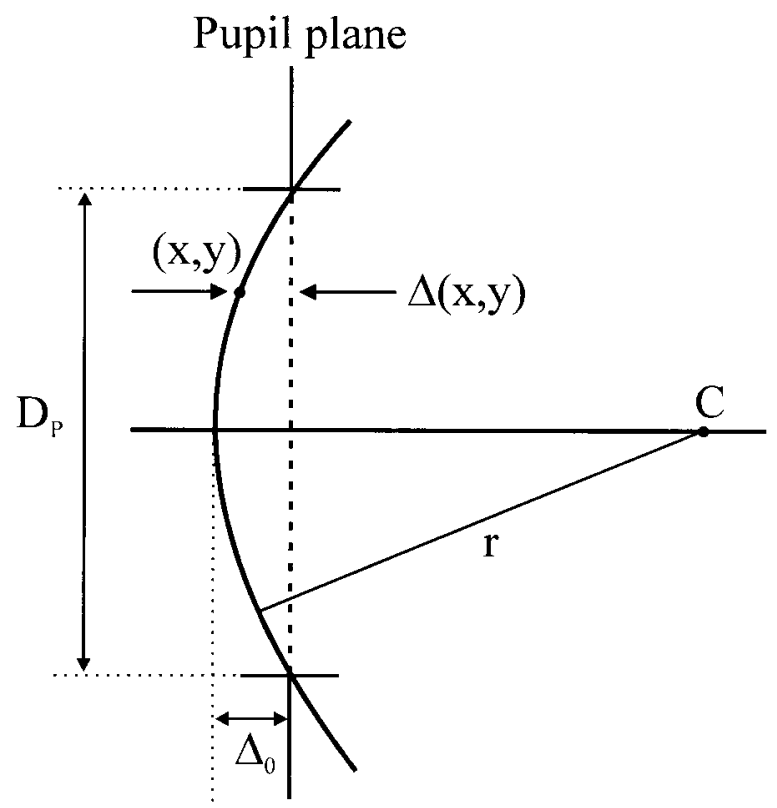

Figure 1. Schematic representation of the human cornea as a diopter.

where $\lambda_{0}$ is the wavelength of the incoming wave in the vacuum, $n$ is the refractive index of the material, the first addend is the phase delay introduced by the diopter and the second addend represents the phase delay introduced by the remaining region of free space between the two planes.

With this, the diopter may be represented as a multiplicative phase transformation of the form

$$
t(x)=\exp \left[i \frac{2 \pi}{\lambda_{0}} \Delta_{0}\right] \exp \left[i \frac{2 \pi}{\lambda_{0}}(n-1) \Delta(x)\right] .
$$

In this expression and in the remainder of the paper we will use $1 \mathrm{D}$ notation. Note that extension to $2 \mathrm{D}$ is straightforward.

Typically, for perfect corneas, the radius of curvature is about $8 \mathrm{~mm}$. Under daylight conditions, the diameter of the entrance pupil is around $4 \mathrm{~mm}$ so that a simple geometric analysis provides that $\Delta_{0} \simeq 0.25 \mathrm{~mm}$. These values confirm that the cornea can be analysed through the method just described. Consequently, the transmittance of the cornea may be represented as a multiplicative phase function in the form of expression (3), only by substituting the values of $\Delta(x)$ by the sagittal depths calculated through equation (1).

With the just described procedure the cornea is transformed into a phase surface which will diffract an incident beam. Thus, calculation of propagated fields coming out from the cornea will help to analyse the performance of the surface and the effect of its irregularities over the position and sharpness of the final image of an aphakic eye. 


\section{Near-field Fresnel distributions}

The Fresnel pattern, $u_{z}\left(x_{z}\right)$ at a distance $z$ of a distribution, $u(x)$, illuminated by a plane wave, is given by

$$
\begin{aligned}
u_{z}\left(x_{z}\right)= & \exp \left(\mathrm{i} \frac{\pi}{\lambda z} x_{z}^{2}\right) \\
& \times \int_{-\infty}^{+\infty}\left[u(x) \exp \left(\mathrm{i} \frac{\pi}{\lambda z} x^{2}\right)\right] \exp \left(-\mathrm{i} \frac{2 \pi}{\lambda z} x x_{z}\right) \mathrm{d} x
\end{aligned}
$$

This expression consists of a Fourier transform of the product of the input and a quadratic phase factor, the whole result also being multiplied by an additional quadratic phase. Equation (4) can be numerically calculated through the discrete Fourier transform (DFT).

As has been stated in the literature $[11,12]$, direct evaluation by DFT of expression (4) does not allow the calculation of near-field patterns. The difficulty comes from the high frequencies of a quadratic phase factor. This problem can be overcome by propagating the angular spectrum. Physically this procedure is equal to propagating the Fourier transform of the signal instead of the signal itself [12]. In this domain equation (4) is transformed into the product

$$
\tilde{u}_{z}(\xi) \propto \widetilde{u_{0}}(\xi) \exp \left(-\mathrm{i} \pi \lambda z \xi^{2}\right)
$$

where the dependence on $z$ of the quadratic phase factor is just the converse than in expression (4).

The near-field calculation algorithm will consist of taking the Fourier transform of the input signal, multiplying it by the sampled version of the quadratic phase factor in equation (5) and then performing an inverse Fourier transform, i.e.

$$
\left(u_{z}\right)_{\mu} \propto \operatorname{DFT}^{-1}\left\{\operatorname{DFT}\left[u_{0}\left(\frac{m \Delta x_{0}}{N}\right)\right] \exp \left(-\mathrm{i} \pi \frac{\lambda z}{\Delta x_{0}^{2}} \tilde{m}^{2}\right)\right\},
$$

where $\Delta x_{0}$ is the sampling extension of the object, $N$ is the total number of samples and $m, \tilde{m}$ and $\mu$ are the samples in object, Fourier and Fresnel domains respectively.

As detailed in [11], the Nyquist sampling condition over the quadratic phase factor will provide the proper sampling condition:

$$
z \leq \frac{\Delta x_{0}^{2}}{\lambda N}
$$

In our particular case, corneal maps obtained through expression (1) are sampled with $N=350$, with a pupil diameter of $4 \mathrm{~mm}$. Considering a wavelength of $633 \mathrm{~nm}$ (He-Ne laser), this method provides well-sampled pattern distributions at a distance $z \lesssim 85 \mathrm{~mm}$.

Considering that normal corneas have a focal length of about $32 \mathrm{~mm}$, the nearfield calculation algorithm is accurate for calculating diffracted patterns from the cornea to a middle range distance.

\section{Image quality parameters}

Evaluation of image quality requires the definition of objective parameters that quantize the appearance of the image. Ideally, the best image which one may consider from a punctual object is its stigmatic image, i.e. a delta function. In a 
more realistic case, good-quality systems will provide a narrow Gaussian-like PSF. When pathological corneas are considered, image planes may appear with background noise, severe asymmetries and, in some cases, secondary peaks. These corneas are not very common, but we find that in these rare cases the definition of a good image plane becomes a subject of great importance.

Independently of the particular optical system that is considered, a good PSF peak will fulfill three conditions: sharpness, symmetry and low energy dispersion. Thus, parameters which evaluate the image quality must consider these factors as functions of the propagated distance. Local extremes (maxima or minima) in these so-defined functions will serve to locate image planes of particular interest. Evolution of each parameter with $z$ will also help to quantify the goodness of the image plane and thus the corneal quality.

In order to minimize the influence of the object position, we will work with collimated beams arriving from infinity. In this case, a perfect system-a normal cornea in our case-working under the paraxial condition will provide a sharp and symmetric peak at the focal plane. This plane will be clearly located by all the quality functions.

In deformed corneas the focal plane is not clearly defined, and thus the extreme of each quality function will not be located at any particular distance. Moreover, extremes for different functions will not always coincide at the same point. Thus, one must take into account several factors. In our case, we will consider the height of the energy peak, the energy dispersion and the symmetry of the light distribution.

Let us consider the normalized distribution of energy at any distance $z$ from the object:

$$
I_{z}\left(x_{z}, y_{z}\right)=\frac{\left|u_{z}\left(x_{z}, y_{z}\right)\right|^{2}}{\int_{A}\left|u_{z}\left(x_{z}, y_{z}\right)\right|^{2} \mathrm{~d} x_{z} \mathrm{~d} y_{z}}
$$

where $A$ is the integration area in the propagated plane.

As a first approximation, the best image plane will be set at the plane with the highest energy peak. This parameter allows comparison between different peak levels for different distances and even for different corneas. Of course, this does not assure that the energy distribution inside the propagation plane is optimum since only one point in the distribution is considered. This means that secondary peaks or asymmetries in the energy distribution will not be detected and other parameters may be considered.

Energy dispersion inside a propagated plane can be quantified through the calculation of the entropy. As has already been mentioned in section 1, entropy will be interpreted as a measure of the energy distribution in the image planes considered. The lowest entropy plane will correspond to the best image plane in the sense of minimal aberrations [14]. In the case of a deformed cornea, this plane will be different for the Gaussian image plane.

Intensity distributions through an image can be interpreted as probability density functions (see equation (8)). Following the definition given in [14], the entropy of an energy distribution in a propagated plane at a distance $z$ is defined as

$$
S_{z}=-\int_{A} I_{z}\left(x_{z}, y_{z}\right) \log \left[I_{z}\left(x_{z}, y_{z}\right)\right] \mathrm{d} x_{z} \mathrm{~d} y_{z},
$$


which can be easily evaluated through numerical calculations.

Again, although minimizing this quality parameter will select image planes with minimum energy dispersion, this does not guarantee that the best image plane will be selected. In the particular case that performance of an astigmatic surface is explored, the entropy will be minimum at the Sturm foci, while the best image plane corresponds to a local maximum between the focal planes [15].

The third parameter, which will be used here, consists of a symmetry parameter. Ideally, the optimum choice for this parameter is the eccentricity of the peak basis. Nevertheless, using this factor will require a huge amount of calculations in order to define the geometrical limits of an energy peak. With the purpose of accelerating the process we have chosen a simple symmetry factor which is convenient for our intentions: let us divide the image plane into four quadrants around the axis. Let $E_{m}$ be the total energy in the $m$ quadrant. The most symmetric plane will correspond to the one where the four quadrants reach the same level of energy, or better, the plane where the energy difference between one plane and the others is minimum. Following this definition, the symmetry factor is defined for each pattern at a distance $z$ as

$$
\sigma=\sum_{m, n=1}^{4}\left|E_{m}-E_{n}\right|_{m \neq n} .
$$

Completely symmetric energy distributions will make this factor zero. Astigmatic surfaces may present a local minimum between the two focal planes. Deformed surfaces may present quite asymmetric distributions, and in those cases the irregularity of the symmetry function indicates the presence of high order aberrations. For normal corneas, the value of this parameter will occur as low values around zero. For deformed corneas, $\sigma$ will locate one or more local minima which may correspond to symmetric distributions which may not correspond to any particular image plane.

The three proposed parameters are complementary. Coincidence between them may serve to locate a good image range or even determine the best image plane. In most of the pathological cases, this will not be possible, and thus comparative studies between several corneas, evolution of the quality parameters with the propagation distance and local coincidences between two of them will serve to determine the vision quality of the subject.

Finally we would like to remark that calculation of the proposed parameters can be performed in a very short time. This fact allows integration of the analysis just described in real-time processes and, consequently, expanding the possibilities of a simple corneal topographic study.

\section{Corneal analysis}

We start our analysis by considering two corneas, one completely regular and the other with an astigmatic deformation. This test is necessary in order to validate the method. Afterwards, we will extend our study to the more difficult case for a keratocone.

Let us consider a normal cornea, which topography is shown in figure 2 . Curvature data, which are codified using colours, are transformed into an exponential phase factor by using the method described in section 2 . The pupil 


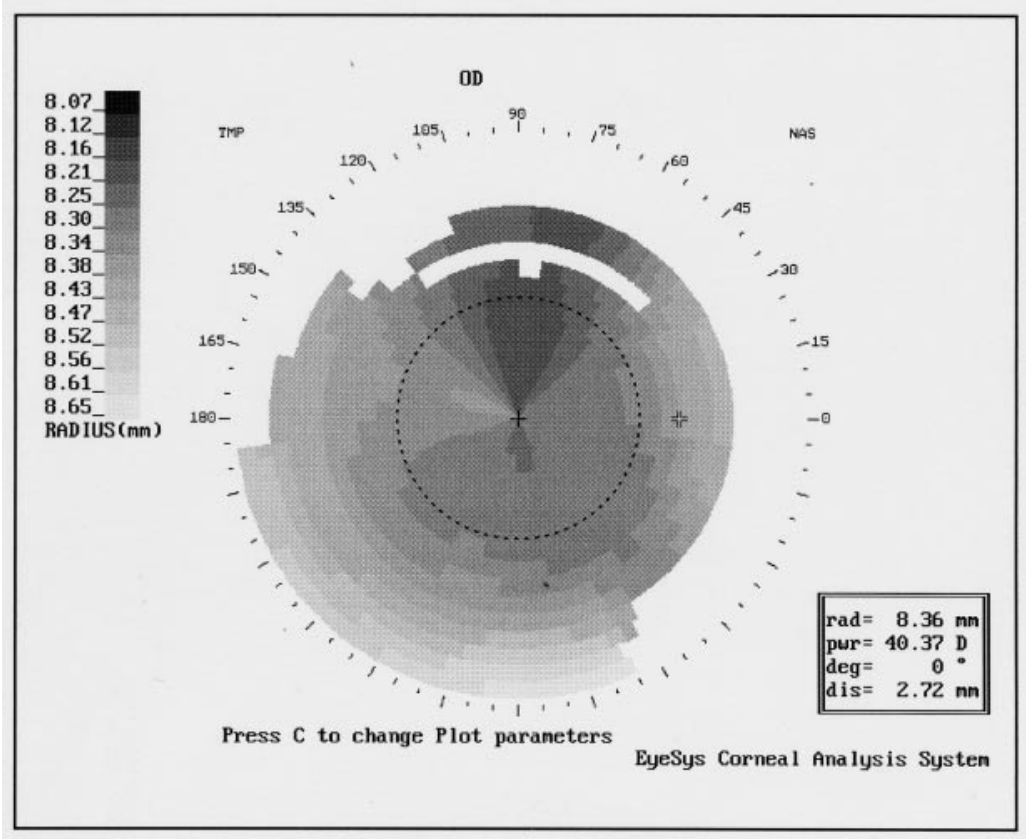

Figure 2. Corneal topographic map of a normal cornea with slight astigmatism. White circumference marks the pupil size which is fixed here.

function so obtained is propagated to a range of distances, and the different quality parameters are evaluated in each plane. In figure 3 we depict the three quality patterns as functions of $z$ for a selected range of distances. The first parameter, the peak height, increases monotonically with the distance of propagation, reaching a maximum at $z=32.2 \mathrm{~mm}$ and then decreases again, as is expected from a regular surface.

Regarding the entropy, this function has a minimum at exactly the same distance $z=32.2 \mathrm{~mm}$. Again, the function is soft and permits defining a point of minimum energy dispersion which coincides with the absolute maximum peak of energy. Regarding the symmetry function, notice that the function takes low values but does not reach zero. This may be interpreted in the sense that the surface which generates these patterns is not perfect but almost spheric-in fact a little astigmatism can be observed in the topography. The most symmetric point corresponds to $z=32.0 \mathrm{~mm}$. Good coincidence of the three extreme points and smoothness of the functions allow us to determine the best image plane at the particular distance already mentioned $(z \simeq 32.1 \mathrm{~mm})$. In figure 4 we represent several propagation planes of size $4 \mathrm{~mm} \times 4 \mathrm{~mm}$, so that one can have a fast visual reference of the corneal quality.

In figure 5 we show an astigmatic cornea. The corresponding quality parameters are shown in figure 6 . It can be observed that the energy reaches two maxima of almost the same value. These distances correspond to the two Sturm focal planes. These planes are also located by the entropy parameter. The local maximum localizes the best image plane between these two foci which also results in the plane with the maximum symmetry $(z \simeq 29.4 \mathrm{~mm})$. Different representative 
(a)

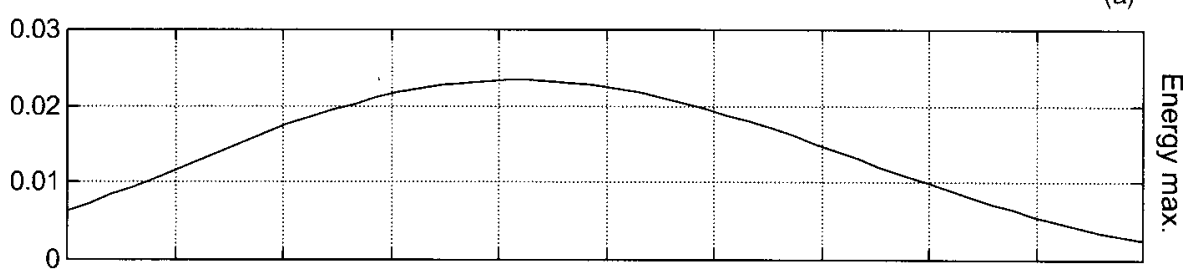

(b)
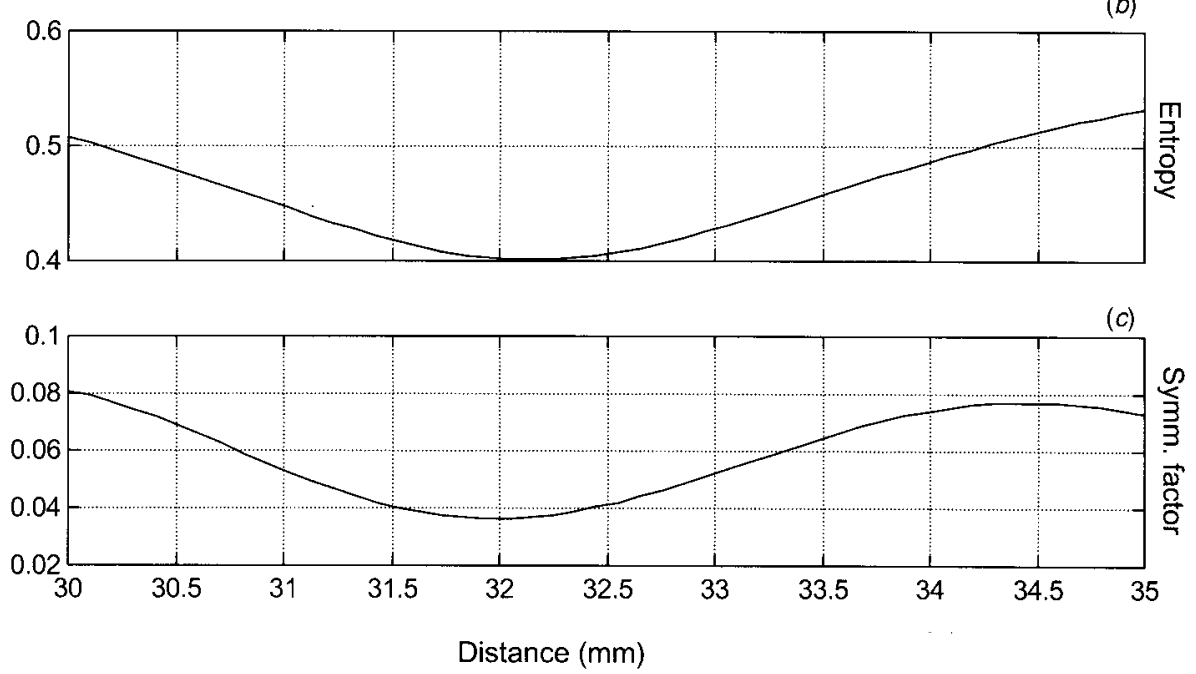

Figure 3. Quality criteria for the cornea depicted in figure 2.

(a)

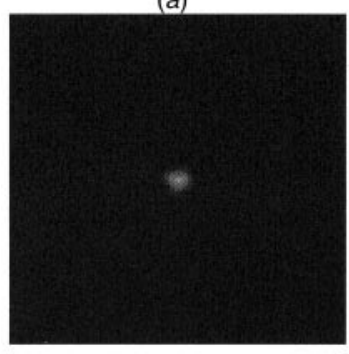

$\mathrm{z}=30.0 \mathrm{~mm}$ (b)

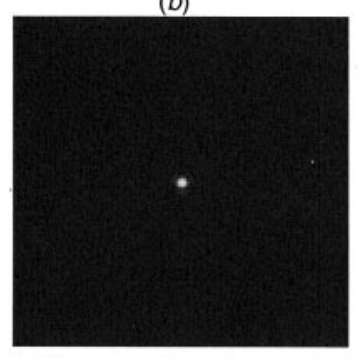

$\mathrm{z}=32.1 \mathrm{~mm}$ (c)

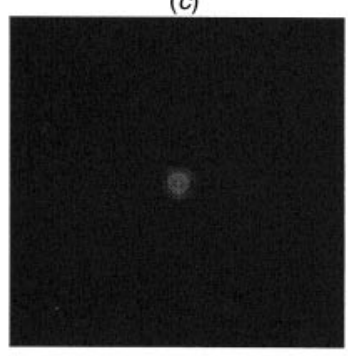

$\mathrm{z}=35.0 \mathrm{~mm}$

Figure 4. Light patterns propagated from the cornea in figure 2. The central distribution corresponds to the best image plane.

propagated planes from this cornea are shown in figure 7 . Again the patterns show a region of about $4 \mathrm{~mm} \times 4 \mathrm{~mm}$.

At this point it is noticeable that the symmetry factor decreases at the borders of the selected range of distances. This effect occurs because unfocused planes consist of symmetric energy distributions with high energy dispersion. Thus, the range of interest must be selected with attention to the other two parameters. Once low dispersion range is selected, the symmetry function estimates the form of the peak. 


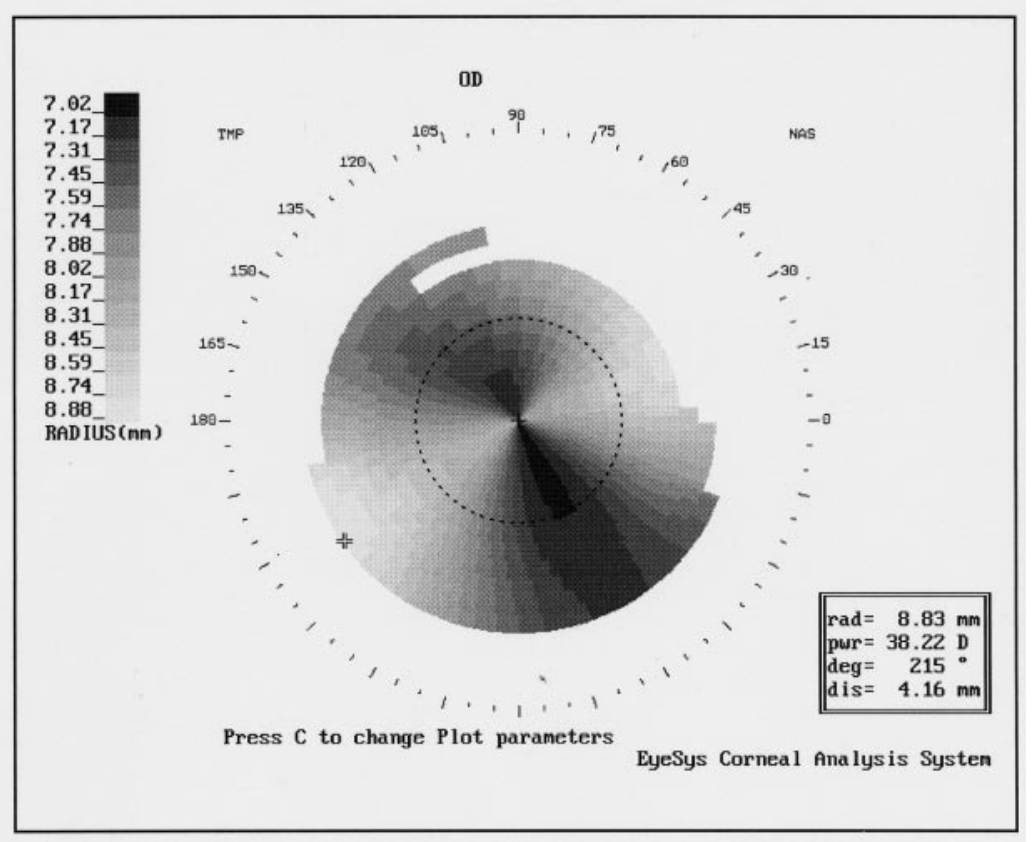

Figure 5. Corneal topographic map of an astigmatic cornea.
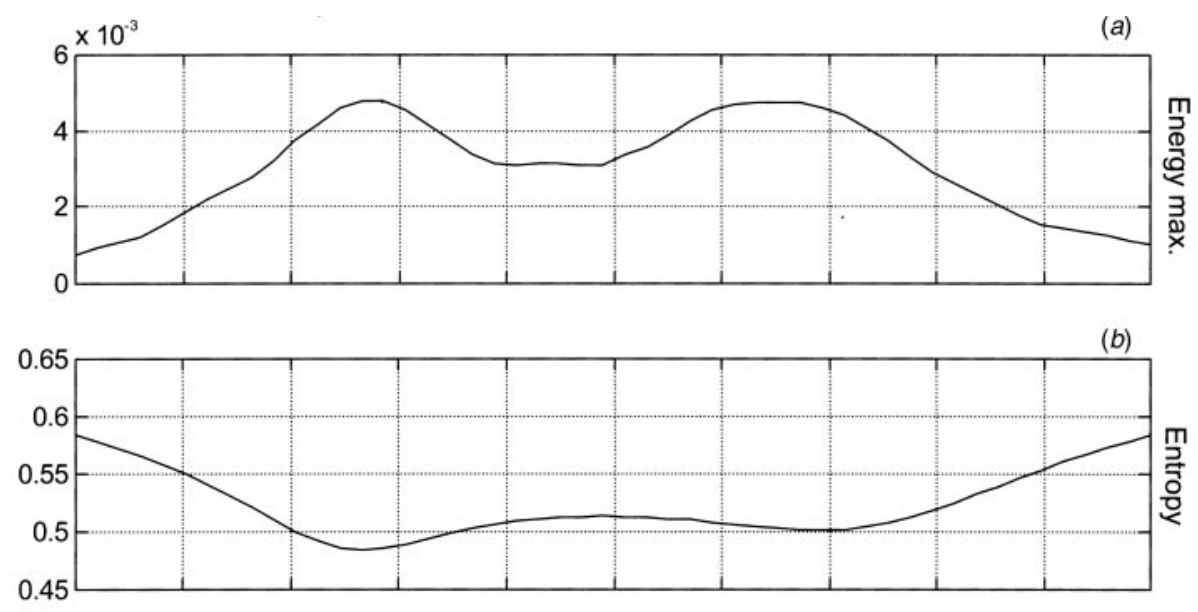

(c)

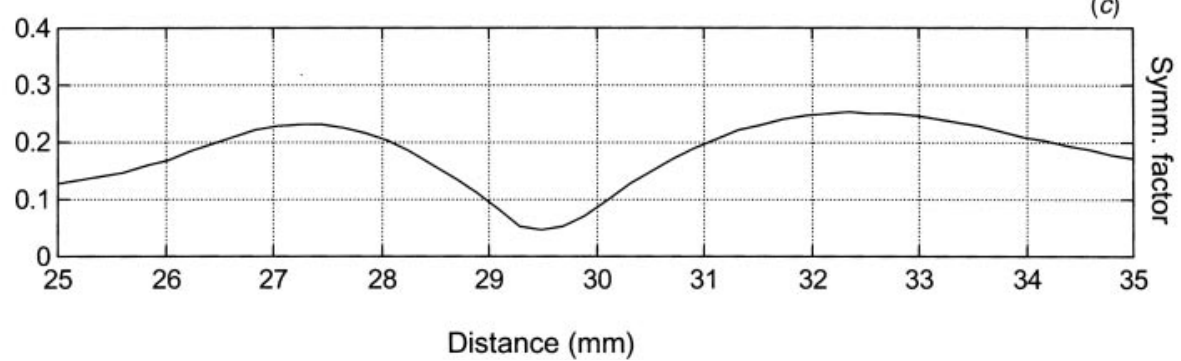

Figure 6. Quality criteria for the cornea depicted in figure 5. 
(a)

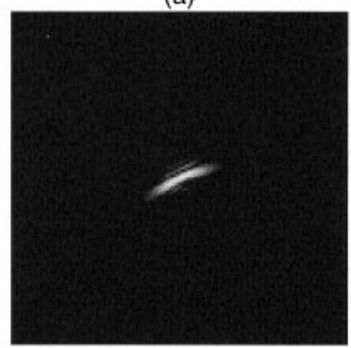

$\mathrm{z}=27.7 \mathrm{~mm}$ (b)

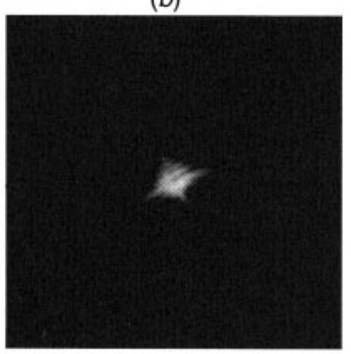

$$
\mathrm{z}=29.4 \mathrm{~mm}
$$

(c)

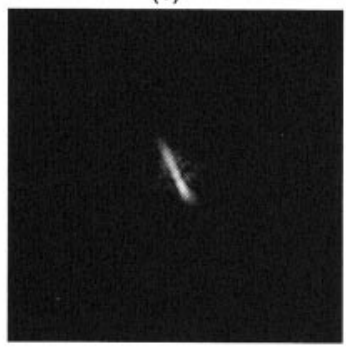

$$
\mathrm{z}=31.9 \mathrm{~mm}
$$

Figure 7. Light patterns propagated from the cornea in figure 5. The central distribution corresponds to the best image plane, while others correspond to the astigmatic focal planes.

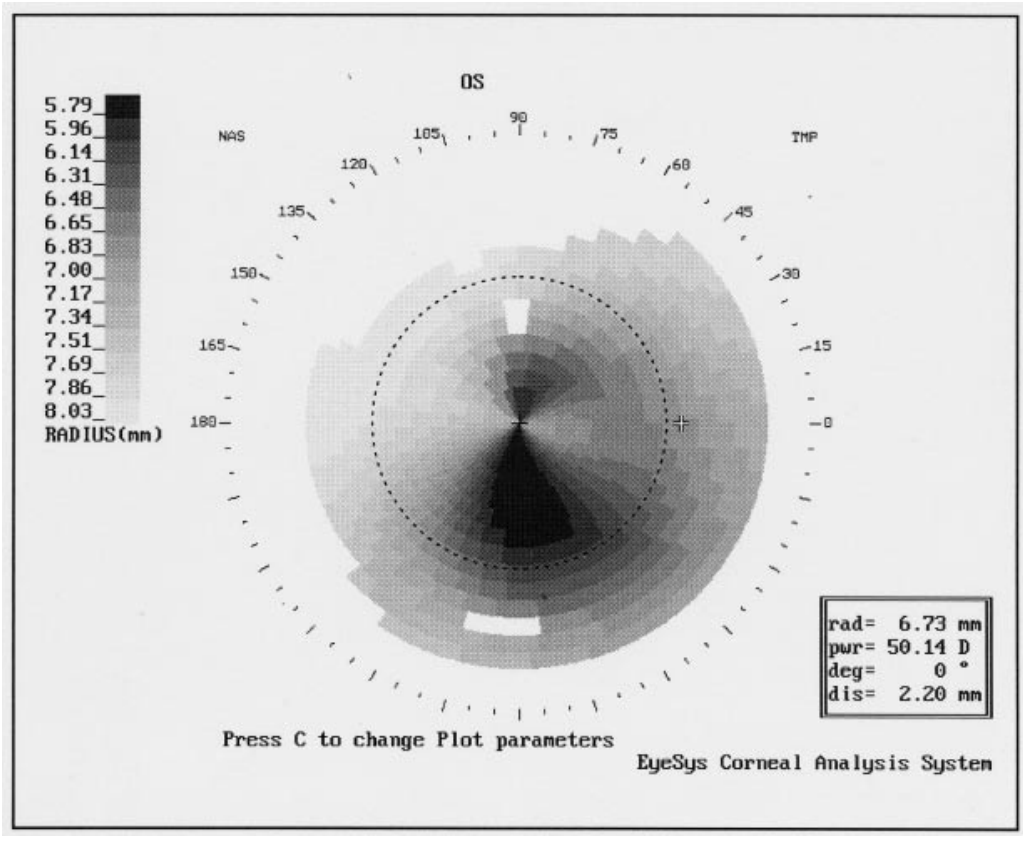

Figure 8. Corneal topographic map of a keratoconus.

Let us consider now a cornea affected by a keratoconus (figure 8 ). The surface of such a cornea is affected by an abnormal growth of the corneal tissue in the lower part of it, producing the effect of a cone. This is usually described as an irregular astigmatism, and in early stages, it can be even mistaken for a normal astigmatism. Optically, a cornea affected by keratoconus can be interpreted as a normal cornea with an additional smaller surface embedded into it. This will result in a highly deformed diopter. Light entering by different zones of the surface will focus at different distances, in the same way that an astigmatic lens or a multifocal lens does, but without any regularity. As we will see below, the final effect on the image will be similar to that produced by the coma aberration, i.e. there will appear a maximum with a comet-like tail which correspond to the unfocused patterns [16]. 


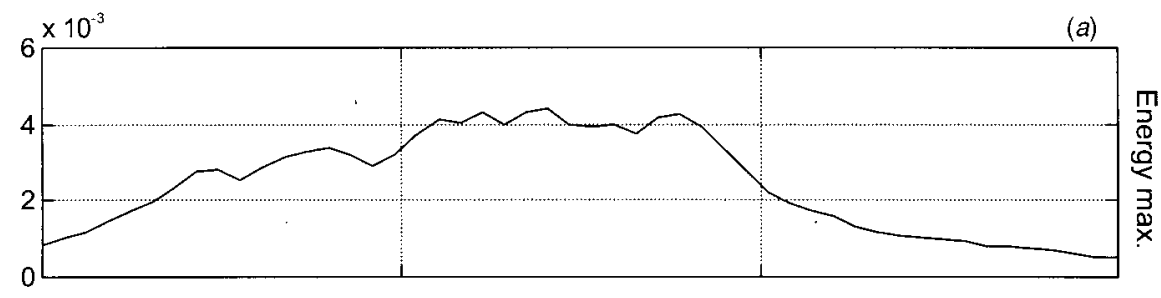

(b)

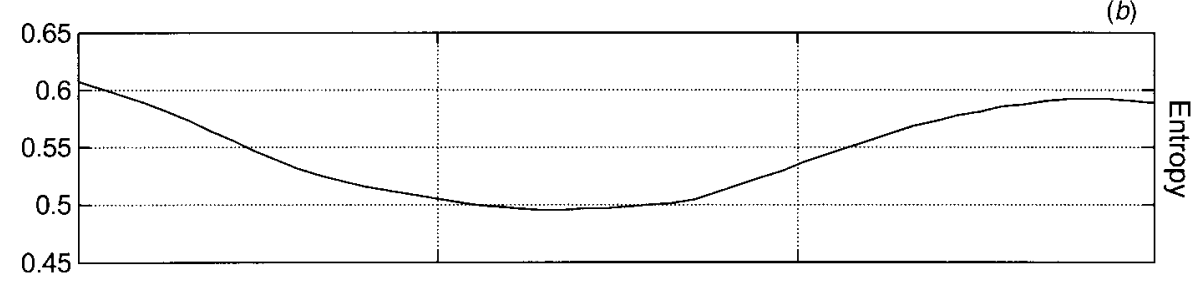

(c)

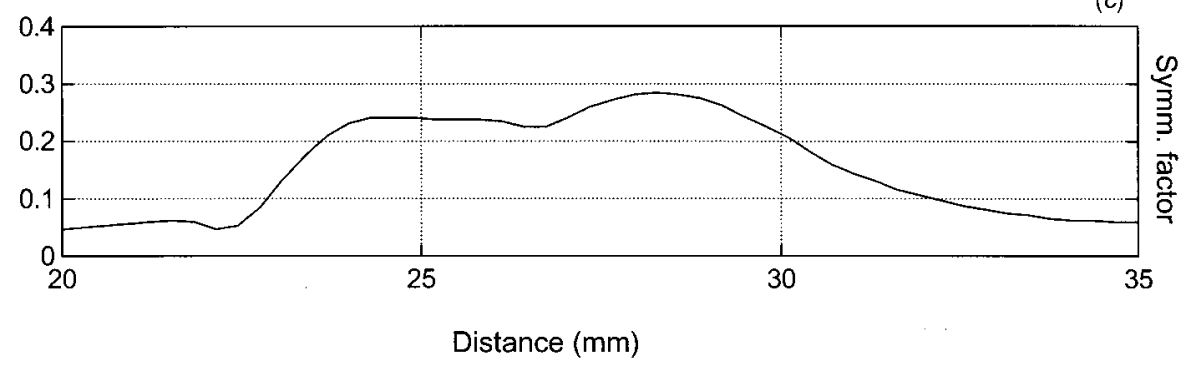

Figure 9. Quality criteria for the cornea depicted in figure 8 .

In figure 9, quality criteria for such a cornea are depicted. The entropy function locates a range of distances where the energy dispersion is low. This limits the wide best-image range. Note that, since the cornea is highly irregular, every part of it focuses at a different distance and several peaks will reach their maxima at different distances.

Regarding the symmetry function, its evolution indicates that the pattern does not properly focus at any distance. We can observe that in the low dispersion range the light patterns present high asymmetries, and thus the quality of vision will be poor, even for this range of distances. A local minima can be found coinciding with a maximum in the energy with the minimum dispersion. In this case, one can fix the vision point at about $26.6 \mathrm{~mm}$ from the surface. The appearance of this pattern can be observed in figure 10 , together with other distributions. It is remarkable that this conus is distinguishable from an astigmatism because of the form of the two first quality criteria. In the case of the symmetry function one recognizes the presence of the two astigmatic foci, whose energy distribution is presented in figure 10. However, in this case the astigmatism is very irregular and, consequently, the two focal planes are not well defined. Notice that the size of the image planes is similar to the previous cases already shown.

It is worth noting that even with poor quality, the selected image point is objectively the best one that the subject can afford, accordingly with the criteria established here. 
(a)

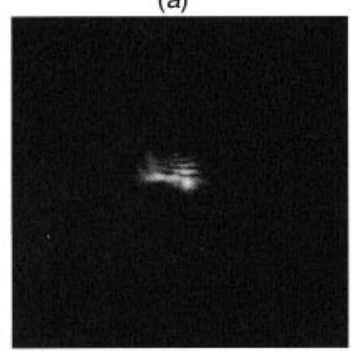

$\mathrm{z}=24.0 \mathrm{~mm}$ (b)

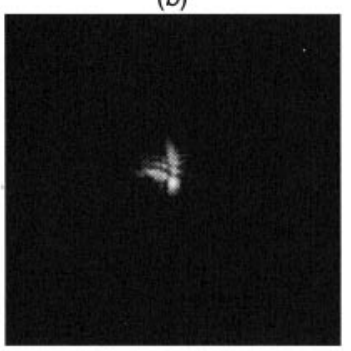

$\mathrm{z}=26.6 \mathrm{~mm}$ (c)

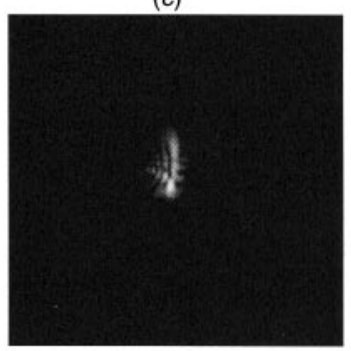

$\mathrm{z}=28.5 \mathrm{~mm}$

Figure 10. Light patterns propagated from the cornea in figure 8. The central distribution corresponds to the best image plane. The other two correspond to astigmatic focal planes.

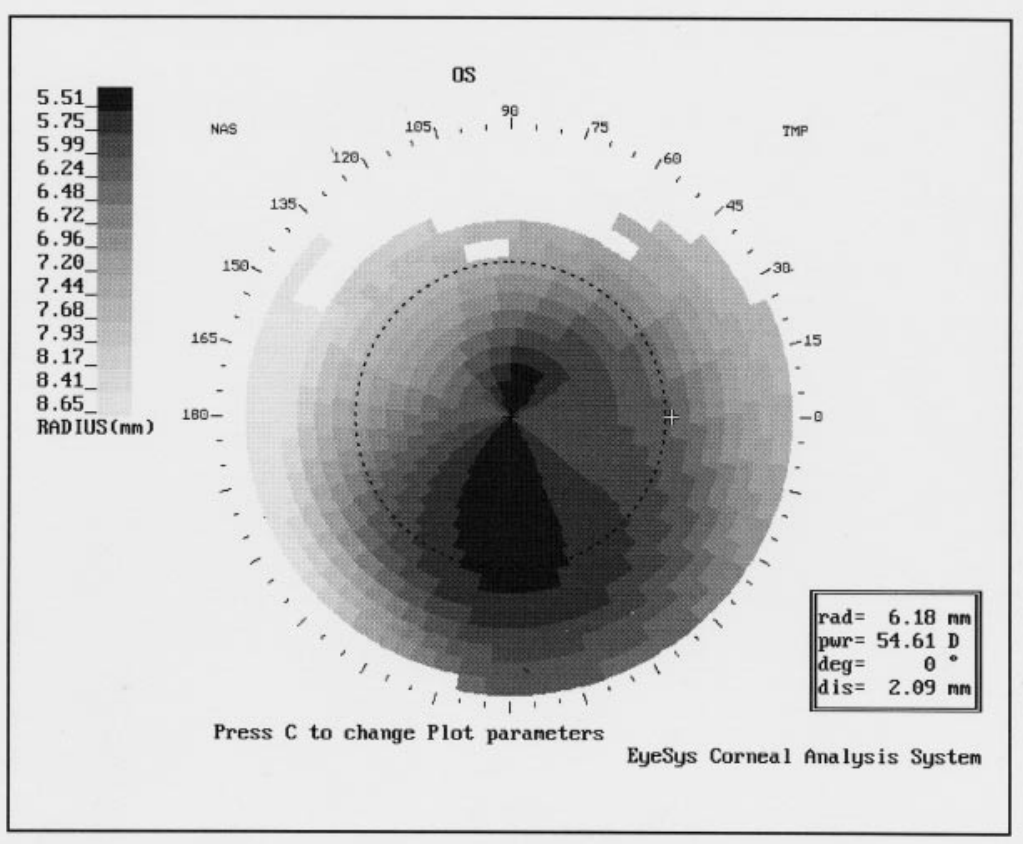

Figure 11. Corneal topographic map of a keratoconus.

Evolution of the quality parameters and propagation planes are very similar in all keratoconus. In figures 11 to 13 we show another example of this pathological case. Note that, again, the entropy determines a range of distances that may be of interest. In this range, the propagated patterns do not focus clearly and, again, a sequence of peaks is obtained. Moreover, the asymmetry of the propagated patterns indicates the presence of severe irregularities in the cornea, and this fact impedes its classification as an astigmatic one. It is also difficult to determine the best image plane, and only a range of distances can be selected according to two of the three criteria. 

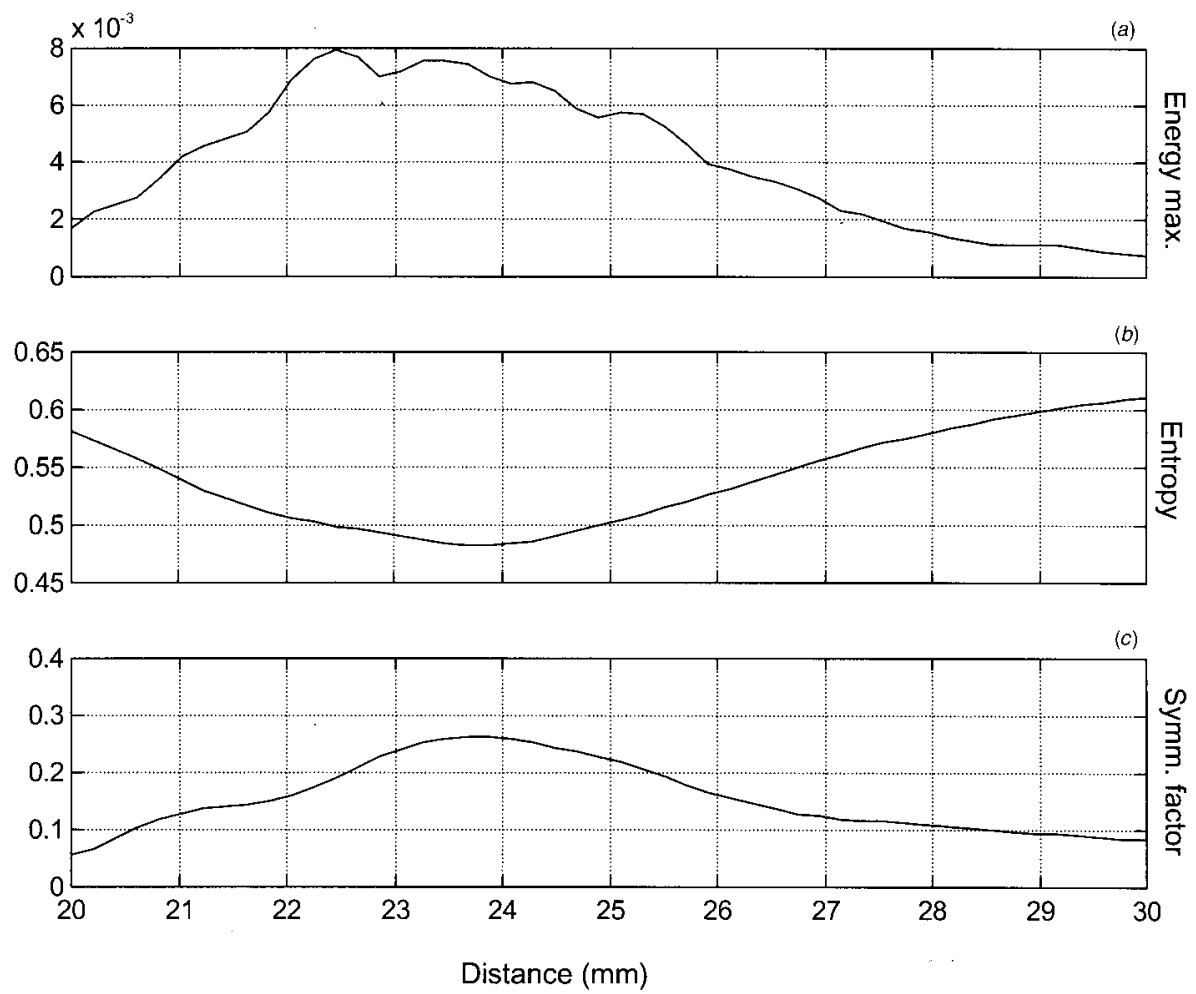

Figure 12. Quality criteria for the cornea depicted in figure 11.

(a)

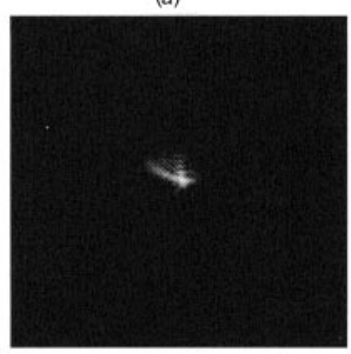

$\mathrm{z}=22.5 \mathrm{~mm}$ (b)

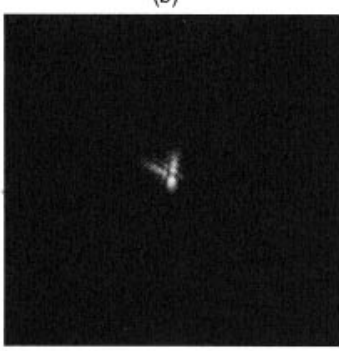

$\mathrm{z}=24.0 \mathrm{~mm}$ (c)

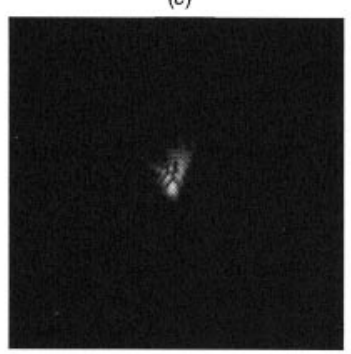

$\mathrm{z}=25.5 \mathrm{~mm}$

Figure 13. Light patterns of size $4 \mathrm{~mm} \times 4 \mathrm{~mm}$ propagated from the cornea in figure 1 . The central distribution corresponds to the best image plane, following only the two first quality criteria (maximum and entropy). The other two distributions do not correspond to any particular plane. The range of distances between them corresponds to the best image range, according to the two first criteria.

Finally in figures 14 to 16 we present an incipient keratoconus. If we first look at the propagated patterns in figure 16 , we can see that these patterns are similar to those obtained for an astigmatic cornea. Let us recall that we are using a pupil of $4 \mathrm{~mm}$ diameter, and thus only the central part of the topography is considered. In this zone, we can see that the topographic map is astigmatic and the conus lies 


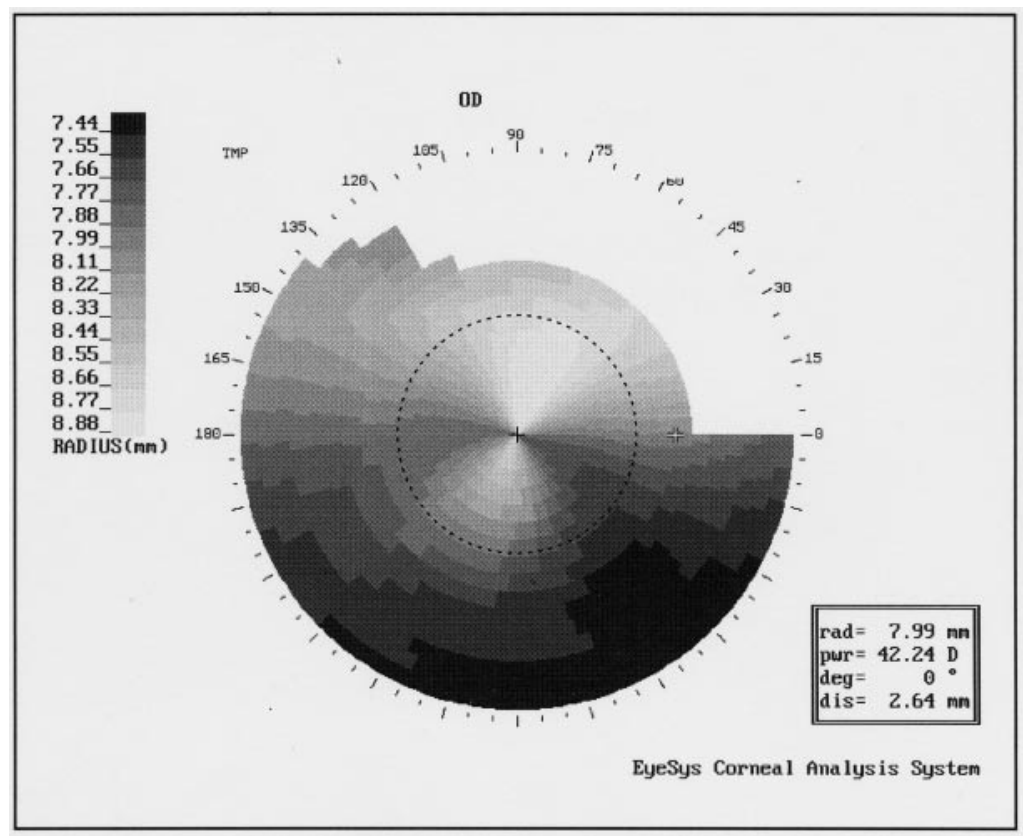

Figure 14. Corneal topographic map of an incipient keratoconus. Notice that the part inside the pupil is similar to the astigmatic case.

outside the considered entrance pupil. Thus astigmatic patterns are obtained. On the other hand, let us consider the evolution of the three quality parameters in figure 15 . In the case of an astigmatic cornea, in figure 6 , we could clearly recognize the presence of two focal planes at different distances. In the case we are now analysing, the presence of different focal planes is not so clear. In the first graph of figure 15, we can recognize the presence of two maxima, but there is no clear decay between them. In the case of the symmetry function, we can see that there is a severe decompensation between the two Sturm planes, just as we found in figure 12 , corresponding to a mature keratoconus. In fact evolution of the three parameters is more similar to that case than to an astigmatism. All these details lead us to classify this cornea as an incipient conus and not as an astigmatism.

\section{Conclusions}

In this paper we have introduced several quality criteria to evaluate the optical performance of an aphakic eye. Corneal data are obtained from topographies of the surface. Heights measured from a reference plane are transformed into differences of optical path and then converted into phase delays.

The calculated corneal function is propagated using a near-field Fresnel diffraction algorithm and different light distributions at different distances are obtained. The appearance of these patterns is evaluated with three quality criteria which measure energy maximum, dispersion of the light and the peak symmetry. Coincidence in all three criteria will locate a best image range or even a best image plane. 

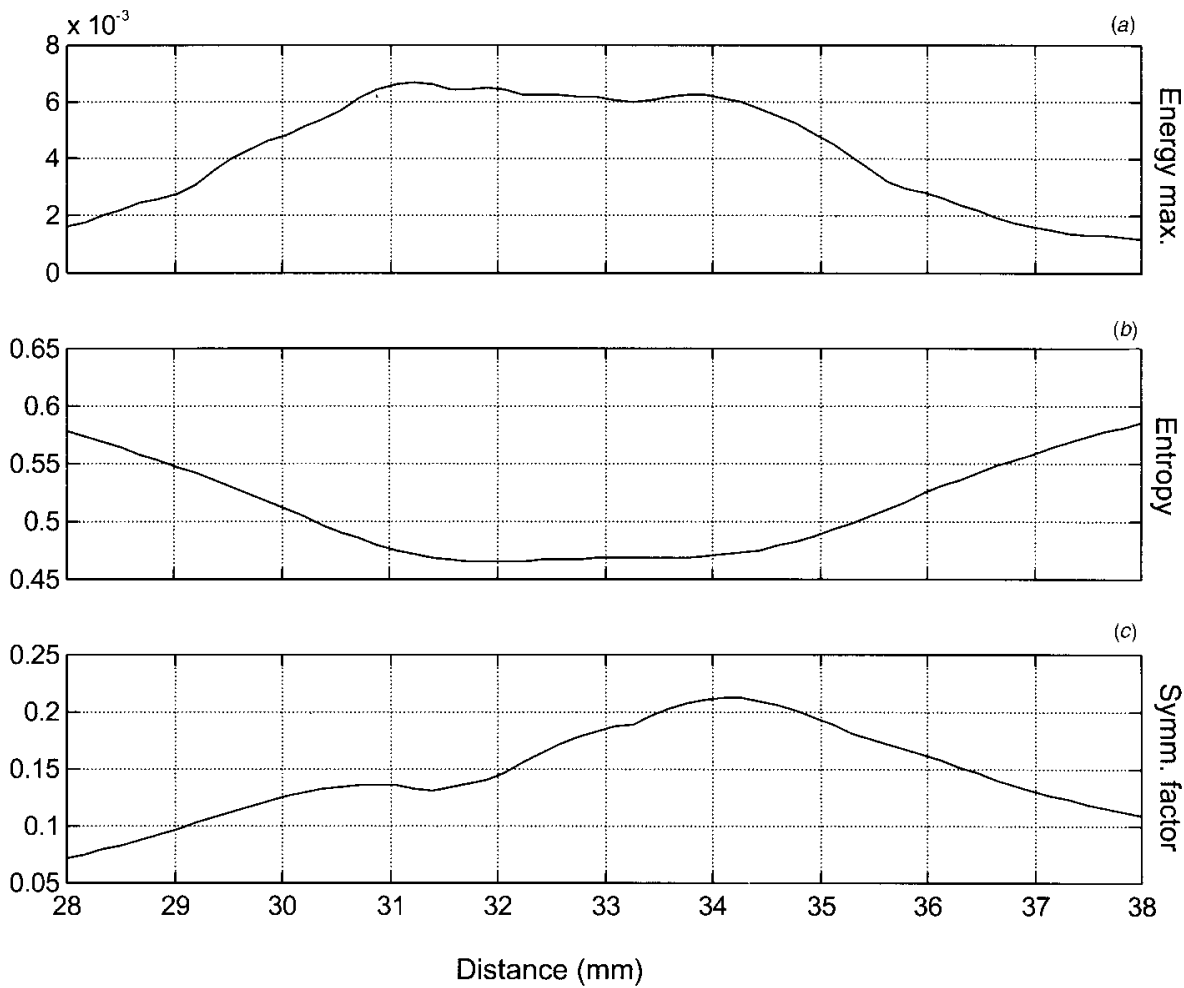

Figure 15. Quality criteria for the cornea depicted in figure 9.

(a)

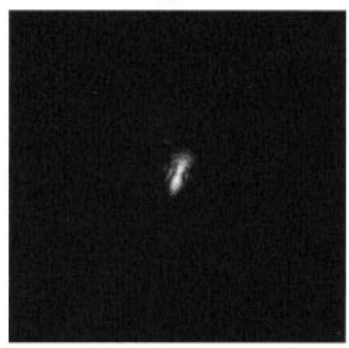

$\mathrm{z}=30.0 \mathrm{~mm}$ (b)

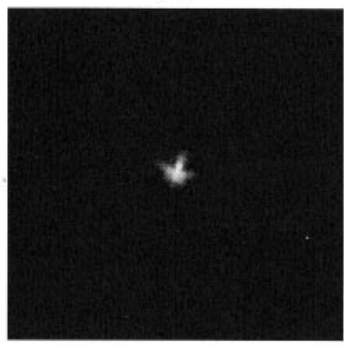

$\mathrm{z}=31.5 \mathrm{~mm}$ (c)

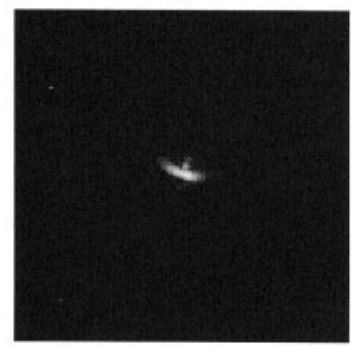

$\mathrm{z}=33.0 \mathrm{~mm}$

Figure 16. Light patterns of size $4 \mathrm{~mm} \times 4 \mathrm{~mm}$ propagated from the cornea in figure 8 . The central distribution corresponds to the best image plane, while the others correspond to the Sturm focal planes.

With the criteria established here, the best image plane is found at the distance where the light distribution becomes as high, narrow and symmetric as possible. In regular corneas working with controlled beams, this plane will coincide, approximately, with the paraxial focal plane. When deformed corneas are used, determination of the theoretical focal plane becomes confused, and therefore the quality criteria may provide very useful information. 
Working with severe malformations in the cornea (keratoconi), we have determined the existence of a range of distances of particular interest. Keratoconi are usually classified as very irregular astigmatic surfaces. The method introduced here permits localization of the irregular astigmatic foci. Prominence of the quality functions with respect to adjacent values helps to estimate the degree of astigmatism which affects the cornea or even, determine if the deformation is completely irregular. In many cases it is also possible to fix a best-image plane which is of particular importance for correcting the defects in the cornea. In any case, the method delimits an image range where the subject affords the best performance that can be obtained without any surgical intervention. It is important to note that all the process can be performed in real time.

Future work must be addressed to incorporate the crystalline lens to the process, in order to obtain the quality function for the whole eye. The technique is especially suitable for studying the optical performance of multifocal intraocular lenses in combination with a real cornea: evolution for the quality parameters will provide very useful information about the quality of each focal plane and may help in producing a personal design for each lens before implanting it inside the eye. With the selection of proper light patterns it is possible to perform computer simulations for several standard opto-types or more complex scenarios.

\section{Acknowledgments}

This work has been supported by the University of Alicante through project number GRE01-09. The authors would also like to thank Mrs Pilar Cacho for her kind collaboration and helpful comments.

\section{References}

[1] Guirao, A., and Artal, P., 2000, J. opt. Soc. Am. A, 6, 955.

[2] Artal, P., and Guirao, A., 1998, Optics Lett., 23, 1713.

[3] Barbero, S., Marcos, S., Merayo-Lloves, J., and Moreno-Barriuso, E., 2002, $\mathcal{F}$. refractive Surgery, 15, 263.

[4] Hemenger, R. P., Tomlinson, A., and Oliver, K., 1995, Ophthalmic physiol. Optics, 15, 63.

[5] Hopkins, H. H., and Yzuel, M. J., 1970, Optica Acta, 17, 157.

[6] Guirao, A., Porter, J., Williams, D. R., and Cox, I. G., 2002, J. opt. Soc. Am. A, 3, 620.

[7] Mahajan, V. N., 1991, Aberration Theory Made Simple (Washington: SPIE Press), p. 75 .

[8] Torroba, R., Rabal, H., and Ruiz, B., 1992, J. mod. Optics, 39, 1939.

[9] Klein, S. A., and Mandell, R. B., 1995, Invest. Ophthalmic visual Sci., 36, 2096.

[10] Goodman, J. W., 1968, Introduction to Fourier Optics (New York: McGraw-Hill), pp. $77-80$.

[11] Mas, D., Garcia, J., Ferreira, C., Bernardo, L. M., and Marinho, F., 1999, Optics Commun., 164, 233.

[12] Mendlovic, D., Zalevsky, Z., and Konforti, N., 1997, J. mod. Optics, 44, 407.

[13] Illueca, C., Mas, D., Perez, J., Pons, A. M., and Artigas, J. M., 2001, J. mod. Optics, $\mathbf{7 8}, 811$.

[14] Carretero, L., Fimia, A., and Beléndez, A., 1994, Optics Lett., 19, 1355.

[15] Carretero, L., Beléndez, A., and Fimia, A., 1996, Appl. Phys. B, 62, 45.

[16] Hemenger, R. P., Tomlinson, A., and Oliver, K., 1996, Ophthalmic physiol. Optics, 16, 124. 